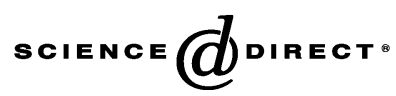

Schizophrenia Research xx (2004) xxx-xxx
SCHIZOPHRENIA

RESEARCH

ELSEVIER

\title{
Executive inhibition and semantic association in schizophrenia
}

\author{
Verity C. Leeson ${ }^{\mathrm{a}}$, Andrew Simpson ${ }^{\mathrm{a}}$, Peter J. McKenna ${ }^{\mathrm{b}}$, Keith R. Laws ${ }^{\mathrm{c}, *}$ \\ ${ }^{a}$ Department of Psychology, London Metropolitan University, London, UK \\ ${ }^{\mathrm{b}}$ Fulbourn Hospital, Cambridge, UK \\ ${ }^{\mathrm{c}}$ Brain and Cognition Research Group, Division of Psychology, Nottingham Trent University, Burton Street, Nottingham, NG1 4BU, UK
}

Received 9 March 2004; received in revised form 20 July 2004; accepted 21 July 2004

\begin{abstract}
Research indicates that some patients with schizophrenia display aberrant inhibition of semantic memory, which may underpin formal thought disorder (FTD). We administered a novel Stroop-like paradigm to three groups of participants: 15 schizophrenic patients with formal thought disorder (FTD), 16 with low FTD ratings, and 15 healthy matched controls. They were required to inhibit a prepotent response for a (previously instructed) required response. Four conditions examined the effect of executive demands by manipulating the relatedness between prepotent and required responses (i.e., identical, semantically related, or unrelated). Two further conditions examining executive function working memory demands required the naming of real or abstract pictures that did and did not necessitate inhibition, respectively. Patients with and without FTD experienced increased difficulty when executive function working memory was required. Moreover, those with FTD also showed increased executive inhibition, but the pattern of errors suggested that the result of this was an automatic activation of semantically related representations. The findings support the notion that increased inhibition underpins the disorganised access to semantic memory in patients with FTD.
\end{abstract}

(C) 2004 Published by Elsevier B.V.

Keywords: Inhibition; Semantic memory; Formal thought disorder

\section{Introduction}

Accumulating evidence points to an impairment of semantic memory in patients with schizophrenia (e.g., Gabrovska et al., 2003; Laws et al., 2000; McKay et al., 1996). Moreover, semantic memory impairment has been be linked to schizophrenic symptoms and, in

* Corresponding author. Tel.: +44 115 8485521; fax: 44115 8486826.

E-mail address: keith.laws@ntu.ac.uk (K.R. Laws). particular, formal thought disorder (FTD), a cardinal symptom of schizophrenia characterised by disturbances in the structure, organisation, and coherence of speech (see metaanalysis by Kerns and Berenbaum, 2002).

Most models of semantic memory assume that semantic knowledge is organised as a network of associated concepts (Collins and Loftus, 1975). Within this framework, concepts are represented as memory nodes, and relations are represented as associative pathways between nodes. Activation in any part of the 
memory network therefore spreads along the associative pathways to related memory areas. Based on network theories of semantics, Maher (1983) proposed that spreading activation may be increased (or inhibition decreased) in schizophrenia, resulting in the intrusion of activated associations into speech which, in turn, manifests as a loss of intelligibility of speech. Others have argued that disorganised activation within the semantic network itself may underlie FTD (Goldberg and Weinberger, 2000; Hoffman, 1987). In contrast to the notion of increased spreading activation, disorganisation reflects failures in activation or increases in inhibition of words or representations closely related in a semantic network, necessitating spread of activation to another more 'distant' sector of a network.

Although inhibition often describes a multitude of cognitive processes, inhibition of prepotent or salient cognitive response has been reported as abnormal in schizophrenia (e.g., Waters et al., 2003). Both disorganisation and increased spreading activation theories of semantic memory in schizophrenia have been proposed as indicators of abnormal inhibition. Nevertheless, whilst the increased activation hypothesis proposes that inhibition is decreased, resulting in spreading of semantic activation to more distantly related semantic nodes in the network, the disorganised activation hypothesis suggests that more distantly related nodes are activated because of an increase in inhibition of closely related nodes.

Increased interference on incongruent trials of the Stroop task has been documented for schizophrenic patients, suggesting that they have greater difficulty inhibiting the prepotent but incorrect response (Barch et al., 1999; Carter et al., 1992). Moreover, Barch et al. (1999) also found this result correlated with disorganised speech symptoms. Nevertheless, others have reported no greater Stroop interference for schizophrenics than controls (Taylor et al., 1996; Jensterle et al., 2000), including two studies of drug-naïve patients (Chen et al., 2001; Carter et al., 1992).

Some inhibitory processes may be linked to executive function. Norman and Shallice (1980) propose that executive function is necessary in tasks that require the subject to overcome strong habitual responses in order to prevent inappropriate responses from gaining control of the action sequence. Thus, the inhibition required in most of the aforementioned tasks and inhibition resulting from context use appear to be executive inhibition. Identifying the nature of inhibition in each experimental paradigm therefore seems essential, because if abnormalities are restricted to subcomponents of the executive system, not all inhibitory mechanisms should be affected. Because executive function working memory may be specifically impaired in schizophrenia and possibly interact with reduced inhibition, the importance of using tasks that can dissociate the various functions is clear. Hence, tasks such as the Wisconsin card sort task can be viewed as tapping working memory, inhibition, and executive function, making it difficult to determine which function(s) is impaired (Laws, 1999).

In a metaanalysis of studies utilising executive function tasks, Kerns and Berenbaum (2002) found that two specific executive functions, inhibition (the suppression of inappropriate thoughts/behaviours) and context memory (maintenance of goal relevant information in performing the task), were significant moderators of the association between FTD and executive task performance. Context memory appears to be analogous to executive function working memory, and the results suggest that the executive system may underlie both deficits.

In summary, equivocal evidence exists for impaired inhibition in schizophrenia and specifically FTD. Inhibition that acts under the control of the central executive in situations that require suppression of a prepotent response has been suggested to be specifically impaired and related to FTD. Furthermore, a second executive function component, executive function working memory, may be impaired in schizophrenia/FTD. This study uses a novel Strooplike paradigm in an attempt to tease apart the relative executive function working memory and executive inhibition problems that may be relate to FTD.

\section{Method}

\subsection{Subjects}

Forty-six subjects participated: 15 schizophrenic patients with high ratings of FTD (defined as a score of $3+$ for global thought disorder derived from all items of the CASH; Andreasen et al., 1992); 16 schizophrenic patients with low ratings of FTD (defined as scoring 0 
2 for global thought disorder on the CASH); and 15 healthy controls. All patients fulfilled the Research Diagnostic Criteria (Spitzer et al., 1978) for schizophrenia. All had normal or corrected-to-normal vision, spoke English as their first language, and had no known history of head injury, neurological disorder, and drug or alcohol misuse. Patients showed a range of illness severity, some were living independently or with minimal support, others lived in sheltered accommodation or in rehabilitation units, and the remainder were chronically hospitalised. The groups were matched (see Table 1) for age, sex, and NART IQ (Nelson, 1982).

\section{Method and procedure}

Six pairs of images were used (see Fig. 1). Each measured $22 \times 15 \mathrm{~cm}$, presented both as single laminated sheets for instruction purposes, and displayed on a laptop computer screen for the reaction time task (using visual basic). On the initiation of each trial (i.e., as the picture was displayed), verbal responses were recorded as a separate sound file on the computer hard drive using the laptop built-in microphone. Each response was later analysed in the acoustic analysis program Soundforge ${ }^{\mathrm{TM}}$, allowing accurate measurement of the latency from image presentation to onset of vocalisation of the response.

In each condition, the two prime pictures were sequentially presented on laminated cards, and participants were taught the required verbal response for each prime. The order of condition presentation was counterbalanced across participants. The computerised version of the task was administered only when subjects had demonstrated that they could generate the correct response to each pair of cards (given the simplicity of the task, the criterion used was $100 \%$ correct score on one occasion); when completed, the next pair was begun and so on for the six pairs. Each condition consisted of two pretest practices and 14 experimental trials. The two pictures were presented in the same pseudorandom order for all subjects and all conditions (AB, BABAABBAABAB). Subjects were immediately informed of incorrect responses and reminded of the required (incorrect) response during practice and experimental trials. Each prime picture was displayed for a maximum of $10 \mathrm{~s}$, with an intertrial interval of $2 \mathrm{~s}$.

The task conditions varied in their demands on executive inhibition and executive function working memory. Four conditions (INH1, INH2, INH3, and INH4) required the inhibition of a prepotent response, naming; the remaining two conditions (NAM and MEM) did not necessitate inhibition. In the case of the NAM condition, this was because the subject was required to name the item portrayed in the image. In the MEM condition, the abstract patterns used were not considered to evoke a name and therefore would not necessitate inhibition of such a name. A previous study using this paradigm in children (Simpson and Riggs, in press) indicated that the required inhibition is greater in the INH1 and INH2 condition (where the prepotent and required responses are from the same set). It is hypothesised that the INH3 condition will also require greater inhibition, because the prepotent responses are semantically related to the incorrect required responses and that this may have a greater impact on the thought-disordered group. Five conditions (INH1, INH2, INH3, INH4, and MEM) placed increased demands on executive function working memory over the naming condition, NAM.

\section{Results}

Mean reaction times for correct responses and numbers of errors in each condition are shown in Table 2. The mean of all conditions requiring

Table 1

Demographic and clinical characteristics of the groups

\begin{tabular}{lllll}
\hline & High FTD & Low FTD & Healthy controls & \\
\hline Age & $49.80(9.43)$ & $41.63(9.95)$ & $42.60(11.12)$ & $F_{2.43}=2.85, \mathrm{~ns}$ \\
NART IQ & $106.53(14.95)$ & $102.63(9.88)$ & $106.53(10.52)$ & $F_{2.43}=0.55, \mathrm{~ns}$ \\
Ratio M/F & $12 / 3$ & $12 / 4$ & $11 / 4$ & - \\
FTD Rating & $3.60(1.02)$ & $0.25(0.68)$ & - & \\
\hline
\end{tabular}

Standard deviations given in parenthesis. 


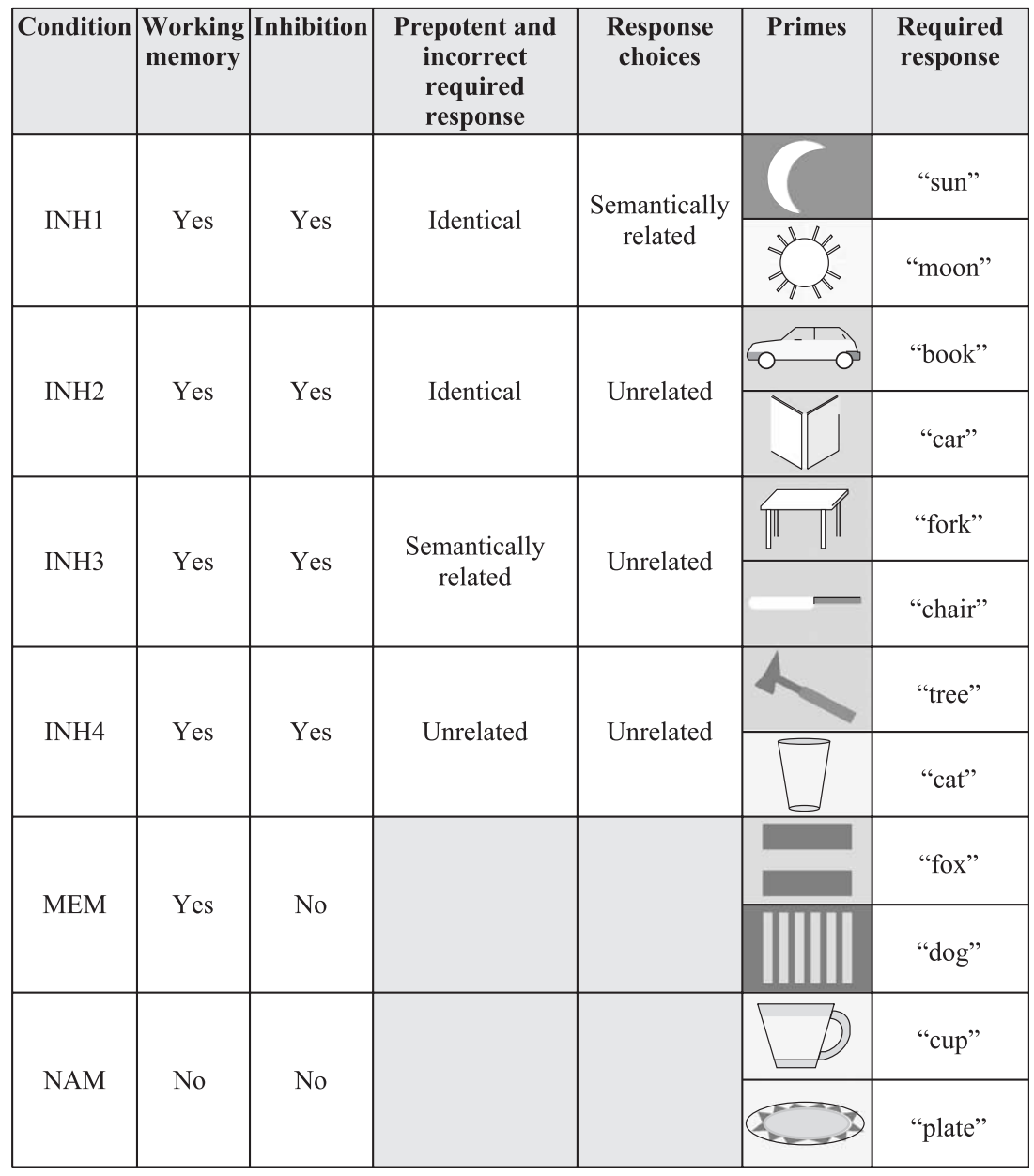

Fig. 1. Primes and required responses for each condition.

inhibition was calculated for each subject (INH1+IN$\mathrm{H} 2+\mathrm{INH} 3+\mathrm{INH} 4)$ and was termed $\Sigma$-INH.

\subsection{Executive function working memory}

\subsection{1. $R T S$}

A mixed ANOVA examining RT was performed with group entered as a between subjects factor (healthy controls, schizophrenics), and memory requirement entered as a within subjects factor (NAM, MEM). This revealed a significant main effect of memory $\left(F_{1,43}=13.06, p<0.001\right)$ and a group-bymemory requirement interaction $\left(F_{1,44}=4.14\right.$, $p<0.05)$. Paired $t$-tests revealed no significant RT increase for controls when working memory was required (NAM vs. MEM, $t_{14}=1.49$, ns), and a significant RT increase when working memory was required both for the low FTD (NAM vs. MEM, $\left.t_{15}=3.41, p<0.01\right)$ and the high-FTD group (NAM vs. MEM, $t_{14}=2.20, p<0.05$ ).

\subsubsection{Errors}

A mixed ANOVA examining errors ${ }^{1}$ with memory (NAM, MEM) as the within subjects factor and group as the between subjects factor (controls, low FTD, high FTD) revealed a significant main effect of

\footnotetext{
${ }^{1}$ Error analyses were repeated using various transformations, but all produced the same outcomes reported here.
} 
Table 2

Mean (standard deviation) reaction times and errors in each condition

\begin{tabular}{|c|c|c|c|c|c|c|c|c|c|}
\hline & \multirow[t]{2}{*}{ Inhibition } & \multirow{2}{*}{$\begin{array}{l}\text { Working } \\
\text { memory }\end{array}$} & \multirow{2}{*}{$\begin{array}{l}\text { Required } \\
\text { response }\end{array}$} & \multicolumn{3}{|l|}{ Mean RTs* } & \multicolumn{3}{|c|}{ Mean errors* } \\
\hline & & & & Controls & Low FTD & High FTD & Controls & Low FTD & High FTD \\
\hline INH1 & Yes & Yes & Identical & $0.70(0.16)$ & $1.04(0.41)$ & $1.26(0.73)$ & $0.95(2.51)$ & $0.00(0.00)$ & $4.37(7.08)$ \\
\hline $\mathrm{INH} 2$ & Yes & Yes & Identical & $0.70(0.11)$ & $1.06(0.42)$ & $1.29(0.57)$ & $0.48(1.84)$ & $0.00(0.00)$ & $2.10(4.55)$ \\
\hline INH3 & Yes & Yes & Semantically related & $0.66(0.11)$ & $1.06(0.38)$ & $1.37(0.58)$ & $1.47(3.04)$ & $4.46(8.99)$ & $18.94(23.98)$ \\
\hline INH4 & Yes & Yes & Semantically unrelated & $0.55(0.09)$ & $0.90(0.29)$ & $1.22(0.55)$ & $0.00(0.00)$ & $0.97(2.65)$ & $11.39(23.25)$ \\
\hline$\Sigma$-INH & Yes & Yes & - & $0.65(0.09)$ & $1.02(0.34)$ & $1.29(0.51)$ & $0.72(0.92)$ & $1.36(0.92)$ & $9.20(13.67)$ \\
\hline MEM & No & Yes & Semantically unrelated & $0.55(0.11)$ & $0.85(0.26)$ & $1.11(0.72)$ & $0.48(1.84)$ & $1.59(3.51)$ & $5.86(8.86)$ \\
\hline NAM & No & No & & $0.52(0.06)$ & $0.69(0.15)$ & $0.87(0.41)$ & $0.00(0.00)$ & $0.48(1.92)$ & $0.48(1.84)$ \\
\hline
\end{tabular}

memory $\left(F_{2,43}=10.22, p<0.01\right)$ and a significant group-by-memory interaction $\left(F_{2,43}=4.44, p<0.05\right)$. Paired $t$-tests showed that only the high-FTD group made significantly more errors in the MEM than NAM conditions $\left(t_{14}=2.60, p<0.05\right)$.

\subsection{Inhibition}

\subsection{1. $R T$}

A mixed ANOVA examining RT with group entered as a between subjects factor (healthy controls, low FTD, and high FTD) and inhibition entered as a within subjects factor (MEM, ¿-INH) revealed a significant main effect for inhibition $\left(F_{1,43}=13.81\right.$, $p<0.001)$ but no significant interaction $\left(F_{2.43}=0.27\right.$, ns). Paired $t$-tests revealed that there was a significant increase in RT when subjects were additionally required to inhibit a prepotent response in the control group (MEM vs. $\Sigma$-INH, $t_{14}=5.15, p<0.001$ ) and in the low-FTD group (MEM vs. $\Sigma$-INH, $t_{15}=2.99, p<0.01$ ). In contrast, the high-FTD group showed no significant increase when inhibition of a prepotent response was additionally required (MEM vs. $\Sigma$-INH, $t_{14}=1.63$, ns).

\subsubsection{Errors}

Paired $t$-tests revealed that errors were not significantly increased in any group when inhibition of a prepotent response was required (MEM vs. $\Sigma$-INH).

\subsection{Level of semantic relatedness}

\subsubsection{RTs}

For the inhibition conditions, no group showed a significant difference in RT or errors when the two required responses were semantically related compared to semantically unrelated (INH1 vs. INH2). RTs were significantly longer when the prepotent and incorrect required response were identical or semantically related than when they were semantically unrelated for the controls (INH1 vs. INH4, $t_{14}=3.96$, $p<0.001$; INH2 vs. INH4, $t_{14}=5.05, p<0.001$; INH3 vs. INH $\left.4, t_{14}=4.03, p<0.001\right)$ and the low-FTD group (INH1 vs. INH4, $t_{15}=2.82, p<0.01$; INH2 vs. INH4, $t_{15}=2.10, p<0.05$; INH3 vs. INH4, $t_{15}=2.61, p<0.05$ ). For the high-FTD group, no significant RT difference occurred between the conditions where the prepotent and incorrect required responses were identical or semantically related and the semantically unrelated condition (INH2 vs. INH4, $t_{14}=0.44$, ns; INH1 vs. $\mathrm{INH} 4, t_{14}=0.25$, ns; INH3 vs. INH4, $t_{14}=1.10$, ns).

\subsubsection{Errors}

Paired $t$-tests showed that the high-FTD group made more errors when the incorrect required responses were semantically related to the prepotent responses compared to when they were semantically unrelated (INH3 vs. INH4: $t_{14}=3.29, p<0.01$ ), but not for the identical conditions compared to the semantically unrelated condition (INH1 vs. INH4: $t_{14}=-1.40$, ns; INH2 vs. INH4: $t_{14}=-1.47$, ns). Neither the controls nor the lowFTD group showed an increase in errors when incorrect required responses were identical or semantically related to the prepotent responses. These differences in errors between the groups across inhibition conditions are shown in Table 3.

\section{Discussion}

Comparisons across conditions revealed that executive function working memory demands had no effect on RT or errors for controls. By contrast however, both 
Table 3

The mean (standard deviation) percentage errors for each group in the four inhibition conditions

\begin{tabular}{llll}
\hline & $\begin{array}{l}\text { Healthy controls } \\
\text { M (S.D.) }\end{array}$ & $\begin{array}{l}\text { Low FTD } \\
\text { M (S.D.) }\end{array}$ & $\begin{array}{l}\text { High FTD } \\
\text { M (S.D.) }\end{array}$ \\
\hline INH1 & $0.95(2.51)$ & $0.00(0.00)$ & $4.37(7.08)$ \\
INH2 & $0.48(1.84)$ & $0.00(0.00)$ & $2.10(4.55)$ \\
INH3 & $1.47(3.04)$ & $4.46(8.99)$ & $18.94(23.98)$ \\
INH4 & $0.00(0.00)$ & $0.97(2.65)$ & $11.39(23.25)$ \\
\hline
\end{tabular}

the high- and the low-FTD groups displayed increased RT, and the high-FTD group showed an increase in errors. Executive inhibition demands increased RT for the controls and the low-FTD group, but not the highFTD group. No group showed an error increase in errors for the inhibition conditions. Furthermore, when the inhibition conditions were examined separately, controls and low-FTD patients were significantly slowed when the prepotent response was identical or semantically related to the incorrect required response compared to when they were unrelated. By contrast, high-FTD patients showed no difference in RT between the identical, semantically related, or semantically unrelated conditions but made significantly more errors in the semantically related condition.

The finding that executive function working memory demands increased RTs in both schizophrenic groups suggests that they found the additional requirements taxing by comparison with the naming condition. Furthermore, the high-FTD patients alone showed increased errors (and slowed responses) for conditions requiring executive function working memory, indicating a specific problem for the highFTD group. It is however important to distinguish between slower RT and errors, despite both being indicative of increased difficulty, because the manifestations of the two in functioning and speech may be different, with trade-offs being informative and sometimes latencies a more subtle indicator of problems than errors (especially on a simple task such as that used in this study).

The pattern of RTs for the controls and low-FTD group in the executive inhibition conditions shows that inhibition of a prepotent response increases task difficulty. Furthermore, both groups showed significantly slowed RTs when the prepotent response was identical or semantically related to the required response (rather than unrelated). This pattern is con- sistent with the notion that activation threshold may be lower (or resting activation is higher) for required responses necessitating greater executive inhibition. Furthermore, the finding that greater executive inhibition is required when the prepotent response is semantically related to the incorrect required response is commensurate with the notion that activation threshold is also lower for nodes neighbouring the required responses. By contrast, high-FTD patients did not evince the increased RT for executive inhibition, and neither the identical nor the semantically related prepotent responses resulted in longer RTs than the unrelated prepotent response. Despite not making more errors on the inhibition conditions, the high-FTD group did show significantly more errors when the prepotent response was semantically related to the incorrect required response. Although the lack of RT increase could be consistent with reduced executive inhibition, the lack of error increase indicates that inhibition was nonetheless effective. Hence, the ability to inhibit a prepotent response seems to be intact in thoughtdisordered patients and may actually be greater than normal (thus resulting in less of an RT increase when inhibition is required compared to low-FTD patients and healthy controls).

Thought-disordered patients showed selective and substantial impairment when the incorrect response was semantically related to the prepotent response, but intact inhibition when the incorrect response was identical to the prepotent response. This suggests that the inhibition of a node results in increased activation for associated nodes. This concurs with Goldberg et al. (2000), who argue that indirect priming reflects failures in activation or increases in inhibition of closely related representations and thus necessitate a spread of activation to a more 'distant' sector of the network. Furthermore, because the high-FTD group did not show an increased RT on this semantically related condition, increased errors appear to reflect a pop-up into the semantic memory of semantically related representation that is not accompanied by doubts over the correctness of the choice.

To conclude, all schizophrenic patients experienced increased difficulty when executive function working memory was required. Nevertheless, this was most pronounced for patients with high-FTD ratings. Furthermore, patients with low-FTD ratings do not differ from healthy controls when executive inhibition 
is required and evince increased RT, particularly if the to-be-inhibited word is either identical or semantically related to the incorrect response. By contrast, highFTD patients show no increase in RT when inhibition is required. Nevertheless, their errors selectively increased when the to-be-inhibited word was semantically related to the incorrect response. This suggests an increase of executive inhibition in FTD that results in an unconscious pop-up of semantically related representations.

\section{References}

Andreasen, N., Flaum, M., Arndt, S., 1992. The comprehensive assessment of schizophrenia symptoms and history (CASH): an instrument for assessing psychopathology and diagnosis. Archives of General Psychiatry 49, 615-623.

Barch, D.M., Carter, C.S., Perlstein, W., Baird, J., Cohen, J.D., Schooler, N., 1999. Increased Stroop facilitation effects in schizophrenia are not due to increased automatic spreading activation. Schizophrenia Research 39, 51-64.

Carter, C.S., Robertson, L.C., Nordahl, T.E., 1992. Abnormal processing of irrelevant information in schizophrenia: selective enhancement of Stroop facilitation. Psychiatry Research 41, $137-146$.

Chen, E.Y.H., Wong, A.W.S., Chen, R.Y.L., Au, J.W.Y., 2001. Stroop interference and facilitation effects in first-episode schizophrenic patients. Schizophrenia Research 48, 29-44.

Collins, A.M., Loftus, A.S., 1975. A spreading activation theory of semantic processing. Psychological Review 82, 407-428.

Gabrovska, V.S., Laws, K.R., Sinclair, J., McKenna, P.J., 2003. Visual object processing in schizophrenia: evidence for an associative agnosic deficit. Schizophrenia Research 59, 277-286.

Goldberg, T.E., Weinberger, D.R., 2000. Thought disorder in schizophrenia: a reappraisal of some older formulations and an overview of some recent studies. Cognitive Neuropsychiatry $5,1-19$.

Goldberg, T.E., Dodge, M., Aloia, M., Egan, M.F., Weinberger, D.R., 2000. Effects of neuroleptic medications on speech disorganisation in schizophrenia: biasing associative networks towards meaning. Psychological Medicine 30, 1123-1130.

Hoffman, R.E., 1987. Computer simulations of neural information processing and the schizophrenia/mania dichotomy. Archives of General Psychiatry 44, 178-187.

Jensterle, J., Mlakar, J., Vodusek, D.B., Frith, C.D., 2000. Disorganisation in schizophrenia need not result from a failure to inhibit dominant response tendencies. Cognitive Neuropsychiatry 5, 105-121.

Kerns, J.K., Berenbaum, H., 2002. Cognitive impairments associated with formal thought disorder in people with schizophrenia. Journal of Abnormal Psychology 111, 211-224.

Laws, K.R., 1999. A meta-analytic review of Wisconsin card sorting studies in schizophrenia: a general intellectual deficit in disguise? Cognitive Neuropsychiatry 4, 1-35.

Laws, K.R., Al-Uzri, M., Mortimer, A.M., 2000. Lexical knowledge degradation in schizophrenia. Schizophrenia Research 45, $123-131$

Maher, B.A., 1983. A tentative theory of schizophrenic utterance. Progress in Experimental Personality Research: Vol. 12. Psychopathology. Academic Press, New York, pp. 1-52.

McKay, A.P., McKenna, P.J., Bentham, P., Mortimer, A.M., Holberry, A., Hodges, J.R., 1996. Semantic memory is impaired in schizophrenia. Biological Psychiatry 139, 929-937.

Nelson, H.E., 1982. The National Adult Reading Test (NART). Windsor, UK.

Norman, D.A. and Shallice, T. (1980). Attention to action: willed and automatic control of behaviour. CHIP Document No. 99. Centre for human information processing, University of California, San Diego, La Jolla.

Simpson, A., and Riggs, K.J. (in press). Inhibitory development in the day-night task and other versions of this developmental Stroop-like task.

Spitzer, R.L., Endicott, J., Robins, E., 1978. Research Diagnostic Criteria: Rationale and reliability. Archives of General Psychiatry $35,773-782$.

Taylor, S.F., Kornblum, S., Tandon, R., 1996. Facilitation and interference of selective attention in schizophrenia. Journal of Psychiatric Research 30, 251-259.

Waters, F.A.V., Badcock, J.C., Maybery, M.T., Michie, P.T., 2003. Inhibition in schizophrenia: association with auditory hallucinations. Schizophrenia Research 62, 275-280. 